This item was submitted to Loughborough's Research Repository by the author.

Items in Figshare are protected by copyright, with all rights reserved, unless otherwise indicated.

\title{
Automatic lane merge based on model predictive control
}

PLEASE CITE THE PUBLISHED VERSION

https://doi.org/10.23919/ICAC50006.2021.9594261

PUBLISHER

IEEE

VERSION

AM (Accepted Manuscript)

\section{PUBLISHER STATEMENT}

(c) 2021 IEEE. Personal use of this material is permitted. Permission from IEEE must be obtained for all other uses, in any current or future media, including reprinting/republishing this material for advertising or promotional purposes, creating new collective works, for resale or redistribution to servers or lists, or reuse of any copyrighted component of this work in other works.

\section{LICENCE}

All Rights Reserved

\section{REPOSITORY RECORD}

Li, Zhaolun, Jingjing Jiang, and Wen-Hua Chen. 2021. "Automatic Lane Merge Based on Model Predictive Control”. Loughborough University. https://hdl.handle.net/2134/16553097.v1. 


\title{
Automatic Lane Merge based on Model Predictive Control
}

\author{
Zhaolun $\mathrm{Li}^{1}$, Jingjing Jiang ${ }^{2}$ and Wen-Hua Chen ${ }^{3}$
}

\begin{abstract}
Autonomous driving has been regarded as the most promising industry since last decade. Among a variety of functionalities an autonomous vehicle has, the automatic merging maneuver is one of the most challenging ones because the maneuver has to be finished in a dynamic traffic environment within limited distance. This paper proposes an integrated path planning and trajectory tracking algorithm based on Model Predictive Control to achieve automatic lane merge in a mixed traffic environment with traditional vehicles (controlled purely by human drivers), semi-autonomous vehicles and fully autonomous vehicles. A bicycle model of vehicle dynamics is used as the prediction model in the algorithm design, while a high-fidelity model with non-linear tyre dynamics is employed in simulation. Moreover, a lane selection function with an add-on threshold function has been used to ensure the safety of the maneuver. The comparison of the simulation results between the proposed algorithm and a bench-marked two-layer control strategy has been given to demonstrate the effectiveness of the proposed controller.
\end{abstract}

Index Terms-Autonomous driving, lane merge, model predictive control, nonlinear vehicle model

\section{INTRODUCTION}

Many functionalities for autonomous vehicles have been studied, such as Adaptive Cruise Control [1], lane keeping assistance [2] and autonomous lane change maneuver [3]-[5]. However, as we know, one main cause for traffic congestion on highways is lane merge and departure. In a traffic environment full of connected and autonomous vehicles, research has been carried out on how to develop cooperative merging strategies. For example, an automated lane merge algorithm based on optimal control has been proposed in [6], in which analytic solutions have been given to reduce computation time, while a control scheme that optimizes all the participants' motion during the merging maneuver has been introduced in [7]. However, the success of such cooperative lane merge was based on the assumption that all vehicles in the merging area follows a high level decision broadcast to all of them. They are designed for the future when all the road users are automated and connected. However, in a foreseeable future,

This work was partially supported by the UK Engineering and Physical Sciences Research Council (EPSRC) Established Career Fellowship (EP/T005734/1).

${ }^{1}$ Zhaolun $\mathrm{Li}$ is with the Department of Aeronautical and Automotive Engineering at Loughborough University, LE11 3TU Loughborough, United Kingdom Z.Lielboro.ac.uk

2 Jingjing Jiang is with the Department of Aeronautical and Automotive Engineering at Loughborough University, LE11 3TU Loughborough, United Kingdom J.Jiang2 a lboro.ac.uk

${ }^{2}$ Wen-Hua Chen is with the Department of Aeronautical and Automotive Engineering at Loughborough University, LE11 3TU Loughborough, United Kingdom W. Chen@lboro.ac.uk it is more likely that vehicles will operate in a mixed traffic environment involving both traditional vehicles and automated vehicles. Hence, this paper studies lane merge in a mixed traffic environment.

Many researchers have been dedicated to solve the automatic lane merge problem and one of the most common way is to build a hierarchical structure which consists of two layers: path planning and trajectory tracking. Many different types of curves have been used to represent the geometry of the path including Nelson polynomials [8], Bezier curves [9], [10], clothoids [11], polynomial spirals [12], spline curves [13], [14] and arcs [15]. Despite the differences between the curves used in all the previous mentioned researches, they are all constructed as solving a two point boundary value problem at each moment [16].

Model Predictive Control (MPC), also regarded as receding horizon control, is a powerful tool to deal with dynamic environment and it was initially used in lane change designs [17], in which it has been used to plan a trajectory for the ego vehicle to place inside the inter-vehicle gap of the target lane. However, if there was no feasible gap, a large amount of computation time would be wasted to searching for it. To tackle this problem, a programmatic approach was proposed in [18] in which an appropriate inter-vehicle gap was determined before lane change planner starting to work. A fully automatic path planning algorithm for lane change was proposed in [19] with a utility function to determine whether a maneuver was desirable. Moreover, a selection function based on reachability analysis was designed to choose the appropriate inter vehicle gap and time instance to initiate the maneuver. However, to reduce the computational load, the prediction model used in [19] is much simpler compared with real vehicle dynamics and a low-level tracking controller is essential. In order to make up for the model mismatch, the constraints were set tighter for both longitudinal and lateral axes to guarantee the safety, which results in a longer maneuver time.

The main contributions of the paper are stated as follows. An MPC based lane merge algorithm is proposed in this paper to achieve automatic lane merge in mixed traffic environment where some vehicles on the road are fully controlled by human drivers and we have no direct control on their behaviour. To simplify the control design and reduce the computational load, the vehicle dynamics is represented as a bicycle model in MPC algorithm development and online implementation, while a high-fidelity model with nonlinear tire dynamics has been used in the simulation to validate the effectiveness of the proposed algorithm. Moreover, an add-on safety threshold function has 
been used to guarantee the safety of the merging maneuver. Finally, the algorithm not only generates a feasible trajectory of the vehicle to follow, but also provides input values that can be applied directly to the vehicle. Thus, it eliminates the model mismatch between the traditional two layers of path planning and trajectory tracking.

The rest of the paper is organized as follows. The bicycle model used in the control design, as well as the high-fidelity model representing the vehicle dynamics in the simulation, is described in Section II, followed by a lane selection function with the add-on threshold in Section III. The automatic lane merge algorithm based on the MPC structure is detailed in Section IV, while the simulation results to demonstrate the effectiveness of the proposed method are given in Section V. In the last section, the conclusions and future work are discussed.

\section{MODEL DESCRIPTION}

For this paper, a bicycle model has been used to predict the behaviour of the ego vehicle (see Fig. 1 from [20]), the

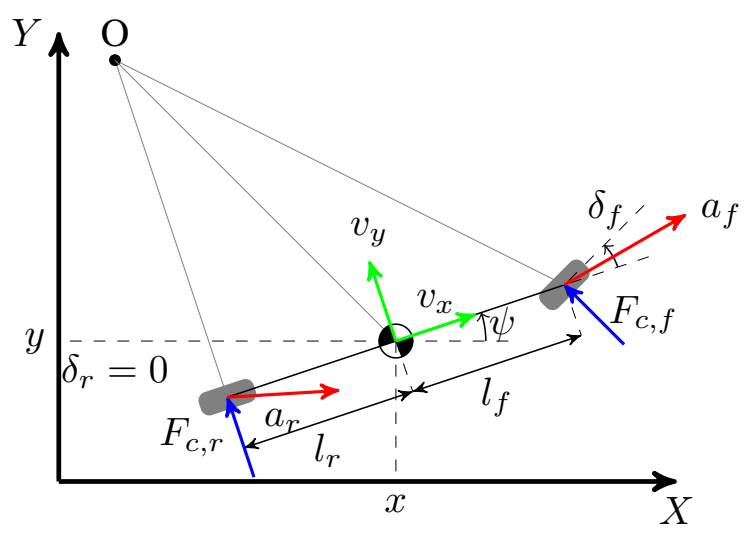

Fig. 1. Dynamic Bicycle Model

dynamics of which is described by the equations

$$
\begin{aligned}
\dot{v}_{x} & =\dot{\psi} v_{y}+a_{x} \\
\dot{v}_{y} & =-\dot{\psi} v_{x}-\frac{2}{m}\left(C_{a, f} a_{f} \cos \delta_{f}+C_{a, r} a_{r}\right), \\
\ddot{\psi} & =\frac{2}{I_{Z}}\left(l_{r} C_{a, r} a_{r}-l_{f} C_{a, f} a_{f}\right), \\
\dot{X} & =v_{x} \cos \psi-v_{y} \sin \psi \\
\dot{Y} & =v_{x} \sin \psi+v_{y} \cos \psi
\end{aligned}
$$

where $v_{x}$ and $v_{y}$ represent the longitudinal and the lateral speed in the vehicle body frame, respectively, while $X$ and $Y$ denote the vehicle's $\mathrm{x}$ and $\mathrm{y}$ position in the global frame, respectively. $\psi, m$ and $I_{Z}$ are the yaw angle, the vehicle mass and yaw inertia, respectively. $l_{f}\left(l_{r}\right)$ describes the distance from the mass center of the vehicle to the front (rear) axle. Apart from that, the cornering stiffness for the front (rear) tyres is represented by $C_{a, f}\left(C_{a, r}\right)$, and $a_{f}\left(a_{r}\right)$ is the slip angle for the front (rear) tyres which is calculated as

$$
\begin{aligned}
& a_{f}=\delta_{f}-\arctan \frac{v_{y}+l_{f} \dot{\psi}}{v_{x}}, \\
& a_{r}=-\arctan \frac{v_{y}-l_{r} \dot{\psi}}{v_{x}}
\end{aligned}
$$

where $\delta_{f}$ is the steering angle and $\dot{\psi}$ represents the yaw rate.

In addition, the high-fidelity nonlinear tyre model used in the simulation is described by (1)-(2) with cornering stiffness $C_{a, f}$ and $C_{a, r}$ replaced by $C_{a, f n}$ and $C_{a, r n}$, respectively, where

$$
\begin{aligned}
C_{a, f n} & =C_{a, f} \frac{a_{o p t, f}^{2}}{a_{o p t, f}^{2}+a_{f}^{2}}, \\
C_{a, r n} & =C_{a, r} \frac{a_{o p t, r}^{2}}{a_{o p t, r}^{2}+a_{r}^{2}},
\end{aligned}
$$

with $a_{\text {opt }, f}=20^{\circ}\left(a_{\text {opt }, r}=11^{\circ}\right)$ [21] is the optimal slip angle for the front (rear) tyres. Note that, the inputs of the system (1)-(2) are the longitudinal acceleration $a_{x}$ at the vehicle body frame and the front wheel steering angle $\delta_{f}$.

Remark 1: For simplicity, double-integrator was used to describe the behaviour of other road users.

\section{LANE SELECTION FUNCTION}

To generate a desirable trajectory for the lane merge scenario, different aspects have to be considered [22]-[24]. Similarly to the utility function in [19], $\mathbb{L}$ is a set containing both merge lane $L_{m}$ and main lane $L_{t}$. The value of the lane selection function $U_{l}$ for Lane $l$, with $l \in \mathbb{L}$, is defined as

$$
U_{l}=w_{1} \frac{U_{l 1}}{N_{1}}+w_{2} \frac{U_{l 2}}{N_{2}}+w_{3} \frac{U_{l 3}}{N_{3}},
$$

where $\omega_{i}>0, i=1,2,3$ are weighting parameters and $N_{1}$, $N_{2}$ and $N_{3}$ are normalization factors calculated as

$$
\begin{aligned}
& N_{1}=\frac{d_{\text {restr }, l}}{v_{\text {main }}}, \\
& N_{2}=\left|\frac{d_{\text {restr }, l}}{v_{\text {main }}}-\frac{d_{\text {restr }, l}}{\gamma_{l}}\right|, \\
& N_{3}=\alpha_{l} g_{\text {rest }, l} .
\end{aligned}
$$

$U_{l 1}$ represents the remaining traveling time defined as

$$
U_{l 1}=\frac{\min \left(d_{\text {restr }, l}, d_{\text {end,l },}-d_{E}\right)}{v_{\text {target }}},
$$

where $d_{e n d, l}-d_{E}$ calculates the distance between the current longitudinal position of the ego vehicle and the end point of merge lane $L_{m}$. In addition, the restriction travel distance $d_{\text {restr }, l}$ is defined as $d_{\text {restr }, l}=\beta_{l} v_{\text {main }}$ with $\beta_{l}>20 s$ a sufficiently large number and $v_{\text {main }}$ as the average velocity of vehicles on the main lane.

Average traveling time $U_{l 2}$ which is defined as:

$$
U_{l 2}=-\left|\frac{d_{\text {restr }, l}}{v_{\text {target }}}-\frac{d_{\text {restr }, l}}{\max \left(\gamma_{l}, v_{l u}\right)}\right|,
$$


where $\gamma_{l}>0$ is a constant parameter. Apart from that, $v_{l u}$ is the average vehicle speed on the lane $L_{l}$.

Time gap density $U_{l 3}$ is defined as:

$$
U_{l 3}=\min \left(\alpha_{l} t_{g_{\text {restr }, l}}, t g_{f}, t g_{r}\right),
$$

where $\alpha_{l}>0$ is a constant parameter, $t g_{\text {restr }, l}$ is the restriction time gap for the ego vehicle $E$. In addition, $t g_{f}\left(t g_{r}\right)$ is inter vehicle time gap between the ego vehicle and its front (rear) vehicle. To improve the safety and guarantee that a lane with small inter-vehicle time gap will never be selected, a novel add-on threshold function $T_{l}$ is introduced in this paper as

$$
T_{l}= \begin{cases}\frac{1}{b_{0}} \min \left(b, b_{0}\right), & \text { if } U_{l 3} \geq 0, \\ 0, & \text { otherwise }\end{cases}
$$

where $b=-\frac{1}{\max \left(1, U_{l 3}+1-t g_{\min , l}\right)}+1, b_{0}=$ $-\frac{1}{\max \left(1, U_{l 3, \text { res }}+1-t g_{\min , l}\right)}+1$ with $U_{l 3, \text { res }}$ and $t g_{\min , l}$ user-selected positive constants.

Therefore, the final lane selection function $U_{\text {lane }}$ used in our algorithm is the integration of the utility function $U_{l}$ introduced in [19] and the safety threshold $T_{l}$, i.e.

$$
U_{\text {lane }}=U_{l} \times T_{l} \text {. }
$$

Remark 2: The extra safety threshold function $T_{l}$ is set to make sure that the value of the overall lane selection function $U_{\text {lane }}$ equals to zero when the gap in the associated lane is not sufficiently large for the vehicle to enter safely. Therefore, only if the value of the lane selection function for the main lane is significantly larger than its counterpart of the merge lane, the lane merge process would be initiated. By doing so, the oscillations in the lane merge process can be effectively eliminated.

\section{MPC Based Automatic Lane Merge Design}

The cost function, the constraints for the vehicle model and the prediction horizon are three essential parts to design a MPC-based algorithm. To guarantee the safety of the lane merge process and to maximize passengers' comfort, they are discussed in details as follows.

\section{A. Cost function}

The cost function for the MPC consists of three parts: reference tracking, $J_{1}$, control effort minimization, $J_{2}$, and comfort maximization, $J_{3}$. They are defined as

$$
\begin{aligned}
& J_{1}=Q_{1} \frac{\sum_{k=1}^{n}\left(v_{x, k}^{r}-v_{x, k}\right)^{2}}{v_{x, k, \max }^{2}}+Q_{2} \frac{\sum_{k=1}^{n}\left(y_{k}^{r}-y_{k}\right)^{2}}{W^{2}}, \\
& J_{2}=R_{1} \frac{\sum_{k=1}^{n} a_{k}^{2}}{a_{k, \text { max }}^{2}}+R_{2} \frac{\sum_{k=1}^{n} \delta_{f, k}^{2}}{\delta_{f, k, \max }^{2}}, \\
& J_{3}=M_{1} \frac{\sum_{k=1}^{n} j_{k}^{2}}{j_{k, \text { max }}^{2}}+M_{2} \frac{\sum_{k=1}^{n} \Delta \delta_{f, k}^{2}}{\Delta \delta_{f, k, \text { max }}^{2}},
\end{aligned}
$$

where $Q_{i}>0, R_{i}>0$ and $M_{i}>0$ are constant weighting parameters, for all $i=1,2 . v_{x, k}^{r}$ and $y_{k}^{r}$ are the references for the longitudinal velocity $v_{x, k}$ and the lateral position $y_{k}$ of the ego vehicle, respectively. $v_{x, k, \max }$ and $W$ denote the maximum longitudinal speed of the ego vehicle and the width of the lane, respectively. $a_{k, \max }$ and $\delta_{f, k, \max }$ describe the maximum values for the two inputs, vehicle acceleration $a_{k}$ and front wheel steering angle $\delta_{f, k}$, respectively. In addition, $j_{k, \max }$ and $\Delta \delta_{f, k, \max }$ are used to represent the maximum allowed values for the longitudinal jerk $j_{k}$ and steering rate $\Delta \delta_{f, k}$, respectively. Note that, $v_{x, \text { max }}^{2}, W^{2}, a_{\text {max }}^{2}, \delta_{f, \max }^{2}, j_{k, \text { max }}^{2}$ and $\Delta \delta_{f, \max }^{2}$ are used to normalize each part. Therefore the cost function of the MPC controller can be defined as

$$
J=J_{1}+J_{2}+J_{3},
$$

where $J_{1}, J_{2}$ and $J_{3}$ are given in (11).

\section{B. Constraints on the vehicle states and the control inputs}

For any autonomous vehicle design, safety requirements are essential and they are modeled as states constraints in this paper. In addition, the inputs to the vehicle, just like the inputs to any other physical systems, have hard constraints due to the physical and power limitations. The details of which are given as follows.

1) The lateral speed should always stay within its limitation $v_{y, \text { limt }}$, which is determined by two components: the lateral speed when the ego vehicle reaches its physical limitation $v_{y, p h y}$ due to tyre adhesion and the lateral speed when the ego vehicle rolls over $v_{y, \text { roll }}$, i.e. $v_{y, \text { limt }}=\min \left(v_{y, p h y}, v_{y, \text { roll }}\right)$.

2) The ego vehicle should always keep enough safety distance from the other road users to avoid potential collision.

3) In lane merge scenario, the ego vehicle should never travel backward, indicating that the longitudinal speed of the ego vehicle should always be larger or equal to zero.

4) Both inputs of the vehicle should be restricted by the power limitation of the ego vehicle and the comfort of the passengers.

5) The ego vehicle should never hit the boundaries of the road, i.e. the lateral position $y_{k}$ needs to be bounded.

To sum up, all the constraints can be described by the following inequalities

$$
\begin{aligned}
& 0 \leqslant v_{x, k} \leqslant v_{x, \max }, \forall k=1,2, \ldots, N, \\
&-v_{y, \text { limt }} \leqslant v_{y, k} \leqslant v_{y, \text { limt }}, \forall k=1,2, \ldots, N, \leqslant x_{k, \text { min }} \leqslant x_{k} \leqslant x_{k, \text { max }}, \forall k=1,2, \ldots, N, \\
& y_{k, \text { min }} \leqslant y_{k} \leqslant y_{k, \max }, \forall k=1,2, \ldots, N, \\
& a_{\text {min }} \leqslant a_{k} \leqslant a_{\max }, \forall k=1,2, \ldots, N, \\
& \delta_{f, \text { min }} \leqslant \delta_{f, k} \leqslant \delta_{f, \max }, \forall k=1,2, \ldots, N,
\end{aligned}
$$

where $N$ denotes the number of predicted time steps and is defined as $N=\frac{T_{\text {predict }}}{\Delta t}$, with $T_{\text {predict }}$ representing the time length for the prediction horizon and $\Delta t$ representing the sampling time. Note that the upper and lower bounds for $v_{x, k}, v_{y, k}, a_{k}$ and $\delta_{f, k}$ are constants for all $k=1,2, \ldots, N$, while the bounds of the longitudinal position $x_{k}$ of the ego 
vehicle is time-varying due to the dynamic traffic environment. The time-varying constraints $x_{\max , k}$ and $x_{\min , k}$ are defined based on which lane the ego vehicle belongs to.

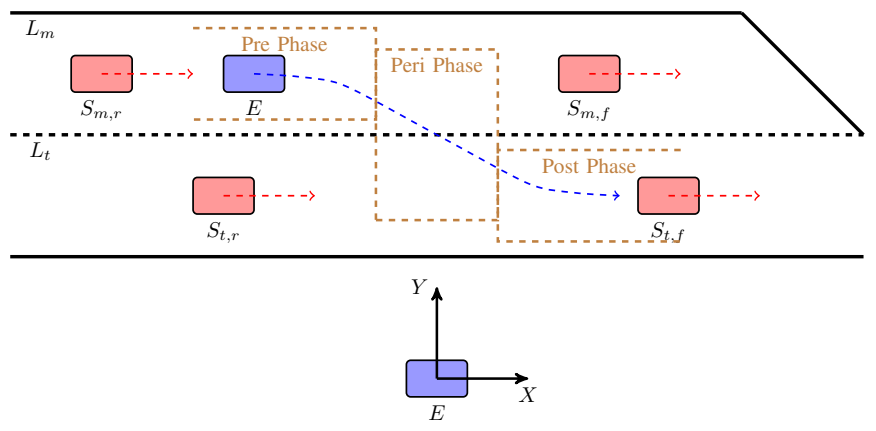

Fig. 2. Two situations of Maneuver

- If the ego vehicle $E$ belongs to a single lane, either the merge lane $L_{m}$ or the main lane $L_{t}$, as denoted by Pre Phase and Post Phase in Fig. 2, it should keep a safe distance from its front vehicle and rear vehicle on that lane, yielding that the upper and lower bounds for the longitudinal position of $E$ are defined as:

$$
\begin{aligned}
& x_{k, \text { max }}=x_{l, f}-s_{m}, \\
& x_{k, \text { min }}=x_{l, r}+s_{m},
\end{aligned}
$$

where $x_{l, f}$ and $x_{l, r}$ are the longitudinal position of the front vehicle $S_{l, f}$ and the rear vehicle $S_{l, r}$ of $E$ on lane $L_{l}$, where $l \in\{m, t\}$. In addition, $s_{m}$ describes the safety margin which is detailed in Remark 3.

- If the ego vehicle $E$ belongs to two lanes as denoted by Peri Phase in Fig. 2, it needs to keep safe distances from all surrounding vehicles in both lanes. This yields that the upper and lower bounds for the longitudinal position for $E$ are defined as

$$
\begin{aligned}
& x_{k, \text { max }}=\min \left(x_{m, f}-s_{m}, x_{t, f}-s_{m}\right), \\
& x_{k, \text { min }}=\max \left(x_{m, r}+s_{m}, x_{t, r}+s_{m}\right) .
\end{aligned}
$$

Remark 3: According to United Kingdom highway driving code rule 126 , there should be 2 s time gap between the two vehicles travel on the highway [25]. Thus, the safety margin $s_{m}$ is present by:

$$
s_{m}=2 v_{S_{i}}+d,
$$

where $v_{S_{i}}$ is the longitudinal velocity of the considered surrounding vehicle and $d$ is a constant safety distance which is set as $3 \mathrm{~m}$ in the simulation.

\section{Prediction Horizon}

Due to restrictions on the communication and cooperation between vehicles in mixed traffic environment, we assume that all the other road users have constant speed in the prediction horizon. Therefore, the prediction horizon should not be too large to capture the dynamics of the traffic. In our design, the prediction horizon is chosen to be $1 \mathrm{~s}$. This also reduces the computational load compared with long prediction horizon.
The dynamics of the other vehicles within the prediction horizon can be described as

$$
\begin{aligned}
x_{S_{i}, k}= & x_{S_{i}, 1}+v_{x, S_{i}, 1} *(k-1) * \Delta t, \\
& \forall k=1, \ldots, \frac{1}{\Delta t},
\end{aligned}
$$

where $x_{S_{i}, 1}$ and $v_{x, S_{i}, 1}$ are the longitudinal position and velocity of the other vehicle participants $S_{i}$. Note that, the position and speed information used in Eq. (17) can be obtained by the sensors on the ego vehicle or through vehicle to infrastructure communication.

Remark 4: The sampling time is set to $\Delta t=0.1 \mathrm{~s}$, so the number of the prediction steps is calculated as $N=\frac{1}{\Delta t}=10$. The surrounding vehicle's states measurements update every $\Delta t=0.1 \mathrm{~s}$ and the prediction horizon is only $1 \mathrm{~s}$, so the prediction of their states is relatively accurate.

\section{Simulation Results}

Matlab function fmincon has been used to solve the MPC problem described in Section IV. A lane merge scenario was designed to test the effectiveness of the proposed algorithm. In addition, a comparison with a hierarchical (high-level path planning and low-level trajectory tracking) control algorithm was also studied. Note that both simulation were carried out with the same constraints, cost functions and parameters.

\section{A. Simulation Set-Up}

TABLE I

CONSTRAINTS AND PARAMETERS USED FOR THE VEHICLE MODEL

\begin{tabular}{|l|l|}
\hline$v_{x} \in\{0,30\} \mathrm{m} / \mathrm{s}$ & $v_{y} \in\{-5,5\} \mathrm{m} / \mathrm{s}$ \\
\hline$a_{x} \in\{-2 \sqrt{2}, 2 \sqrt{2}\} \mathrm{m} / \mathrm{s}^{2}$ & $\delta_{f} \in\left\{-\frac{\pi}{6}, \frac{\pi}{6}\right\} \mathrm{rad}$ \\
\hline$m=1575 \mathrm{~kg}$ & $I_{Z}=2875 \mathrm{~kg} * \mathrm{~m}^{2}$ \\
\hline$C_{a, f}=-25000 \mathrm{~N} / \mathrm{rad}$ & $C_{a, r}=-50000 \mathrm{~N} / \mathrm{rad}$ \\
\hline$l_{f}=1.2 \mathrm{~m}$ & $l_{r}=1.6 \mathrm{~m}$ \\
\hline$W=3.5 \mathrm{~m}$ & $y_{k} \in\{-3.5,3.5\} \mathrm{m}$ \\
\hline$a_{o p t, f}=20^{\circ}$ & $a_{o p t, r}=11^{\circ}$ \\
\hline$\epsilon=1.4$ & $d=3$ \\
\hline
\end{tabular}

TABLE II

PARAMETERS FOR THE LANE SELECTION FUNCTION

\begin{tabular}{|l|l|}
\hline$\alpha_{l}=2$ & $\beta_{l}=30 \mathrm{~s}$ \\
\hline$\gamma_{l}=2$ & $\zeta=0.1$ \\
\hline$v_{\text {target }}=30 \mathrm{~m} / \mathrm{s}$ & $t_{\text {restr }, l}=1.5 \mathrm{~s}$ \\
\hline$\xi=0.01$ & $U_{\text {l1,rest }}=1.7 \mathrm{~s}$ \\
\hline
\end{tabular}

The parameters and constraints related to the ego vehicle and the design parameters for the lane selection functions are shown in Table I and Table II, respectively. Note that we assumed all the vehicles in this lane merge scenario were rectangular with $3 \mathrm{~m}$ length and $2.4 \mathrm{~m}$ width.

In the simulation (shown in Fig. 3), we assume that the ego vehicle $E$ was placed at the merge lane $L_{m}$ with an initial velocity of $15 \mathrm{~m} / \mathrm{s}$ and there are three vehicles on the main lane $L_{t}$ with the initial speed of $21 \mathrm{~m} / \mathrm{s}$. The ego vehicle is aiming to merge into the main lane $L_{m}$. However, after the simulation scenario started, the road user $S_{2}$ decided to accelerate and 
close the gap $G_{A}$. Note that there is an end point for the merge lane which is $250 \mathrm{~m}$ ahead of the ego vehicle's initial position. In addition, the initial states $\left(X, Y, v_{x}, v_{y}\right)$ for Vehicles $E, S_{1}, S_{2}, S_{3}$ are given as $(0,1.75,15,0),(55,-1.75,21,0)$, $(-55,-1.75,21,0)$ and $(-160,-1.75,21,0)$, respectively.

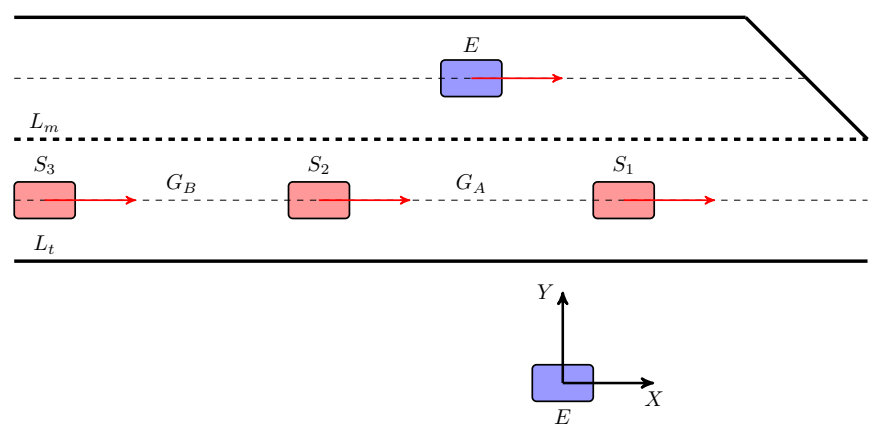

Fig. 3. Lane Merge Scenario

B. Comparison Between the Proposed Algorithm and Benchmarked Two-Layer Control Strategy
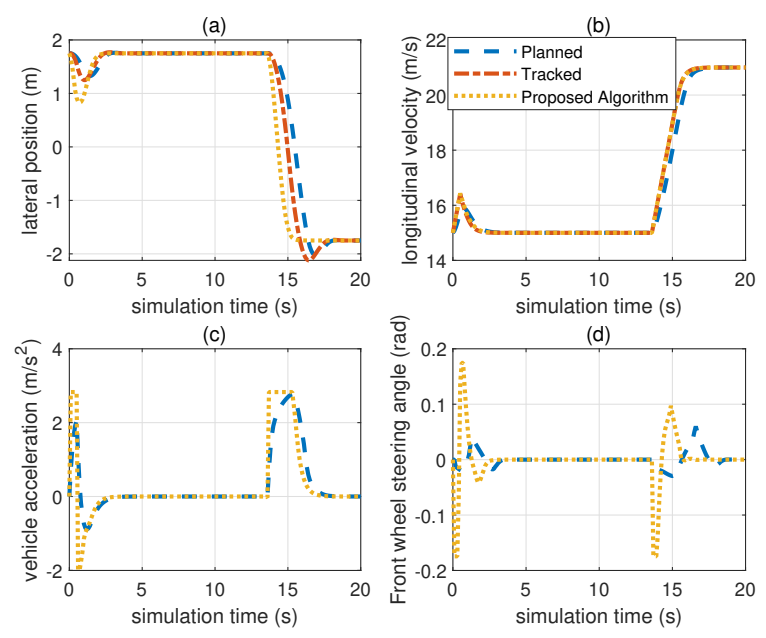

Fig. 4. States of the ego vehicle in Lane Merge Scenario

Simulation results for the lane merge with our proposed MPC based algorithm is shown in Figs. 4 (yellow-dotted line) and 5. From Fig. 4-(a), we can see that the ego vehicle initiated the lane merge maneuver at the beginning of the traffic scenario and attempted to merge into the gap $G_{A}$. However, due to the acceleration of $S_{2}$, the gap $G_{A}$ became unsafe for the ego vehicle to merge into. Hence, the ego vehicle was guided back to the merge lane $L_{m}$ as $U_{m}>U_{t}$ and remained there waiting for another opportunity to merge into the main lane. When $\mathrm{t}=13.5 \mathrm{~s}$, the ego vehicle has detected that it was safe to merge into the gap $G_{B}$ and $U_{t}$ became larger than $U_{m}$. Thus, the lane merge process was re-initiated automatically by the proposed algorithm. Apart from that, the ego vehicle entered the main lane at $0.3 \mathrm{~s}$ and fully went back to the merge lane at $1.3 \mathrm{~s}$ in its first merge attempt. In addition,

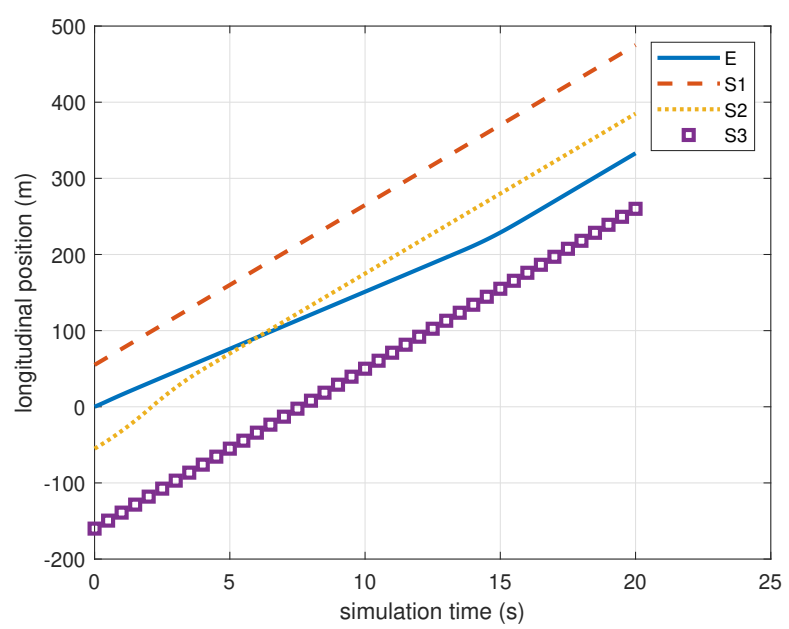

Fig. 5. The longitudinal position of the vehicles in Lane Merge Scenario

in its second merge attempt, the ego vehicle entered the main lane from 13.9s and fully merged into the main lane at $14.8 \mathrm{~s}$. Combined it with the information illustrated in Fig. 5, it is clear that in both merge attempts, i.e. from $0.3 \mathrm{~s}$ to $1.3 \mathrm{~s}$ and $13.9 \mathrm{~s}$ to $14.8 \mathrm{~s}$, the ego vehicle has kept a $2 \mathrm{~s}$ time gap between its front and rear vehicles on the main lane $\left(S_{1}\right.$ and $S_{2}$ for the first and $S_{2}$ and $S_{3}$ for the second). The control inputs of the system are shown in Fig. 4-(c) and (d), from which we can see that none of the inputs has violated the constraints.

In comparison, the simulation results from the two layer hierarchy structure are presented by the orange, dash-dotted lines and blue, dashed lines in Fig. 4. The high level planner has used two independent double integrator as its prediction model [19], while a low-level MPC based controller has been used to track the planned path. From Fig 4-(a) and Fig 4-(b), it is obvious that there is overshot for the lateral position of the ego vehicle in the end of the lane merge process. In addition, we can clearly see the difference between the reference path generated by the planner and the actual trajectory generated by the tracker because the planner only used a simplified vehicle model. Moreover, to complement the model mismatch and to guarantee the safety, tightened constraints have been used by the planner. Hence, the lane merge time has been increased by $72 \%$ (from $2.5 \mathrm{~s}$ to $4.3 \mathrm{~s}$ ).

\section{Effect Analysis of the Add-on Threshold Function}

In this subsection, we study the effects of the threshold function (from $\mathrm{Eq}$ (9)) on the behaviour of the ego vehicle, the results of which are shown in Fig. 6. It can be seen that the lane merge maneuver can not be completed safely without the threshold function. The algorithm stopped at $0.5 \mathrm{~s}$ because it predicted that the longitudinal position of the ego vehicle $x_{k}$ would violate its lower bound $x_{k, \min }$ within $1 \mathrm{~s}$ prediction horizon for all feasible control inputs due to the shrinking of the distance between $S_{2}$ and $E$, while the main lane is still the selected lane as $U_{t}>U_{m}$. However with the threshold function, the ego vehicle was guided back to the merge lane 

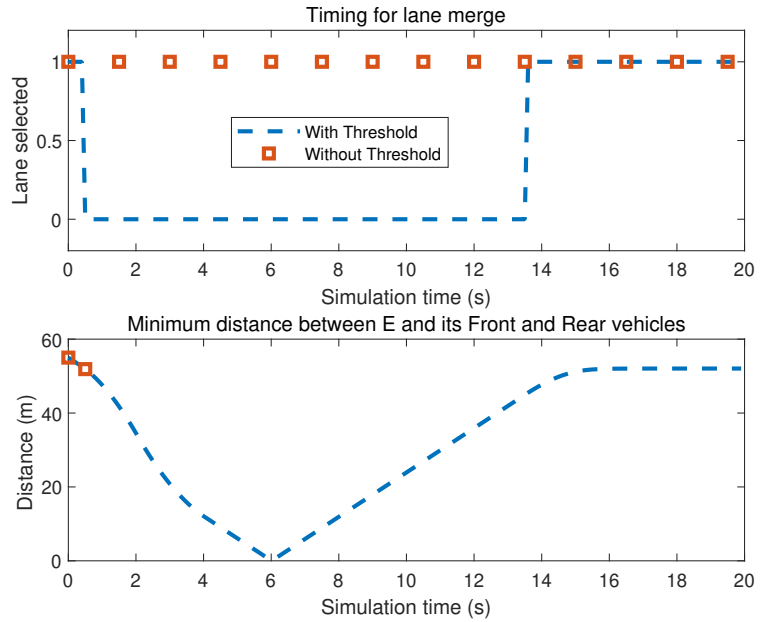

Fig. 6. Comparison between of the lane merge timing with and without the threshold function ( 0 represents merge lane and 1 represents main lane in the first graph)

at $0.4 \mathrm{~s}$ before the gap $G_{A}$ was deemed to be unsafe for the lane merge to continue.

\section{CONCLUSions AND Future Work}

This paper proposed an MPC-based automatic lane merging algorithm for mixed traffic environment with both traditional vehicles and automated vehicles. A nonlinear bicycle model integrating the longitudinal and lateral dynamics of the vehicle has been considered in the algorithm. In addition, a novel lane selection function with add-on threshold has been used to improve the safety. The proposed integrated algorithm has been compared with the widely-used two layer strategy (highlevel path planning and low-level trajectory tracking) and the simulation results show the benefits of adopting our proposed algorithm. In the future, we will study how to extend the algorithm to other driving scenarios, such as overtaking.

\section{REFERENCES}

[1] S. E. Shladover, C. Nowakowski, X.-Y. Lu, and R. Ferlis, "Cooperative adaptive cruise control: Definitions and operating concepts," Transportation Research Record, vol. 2489, no. 1, pp. 145-152, 2015.

[2] X. Xu, J. W. Grizzle, P. Tabuada, and A. D. Ames, "Correctness guarantees for the composition of lane keeping and adaptive cruise control," IEEE Transactions on Automation Science and Engineering, vol. 15, no. 3, pp. 1216-1229, 2017.

[3] Y. Luo, Y. Xiang, K. Cao, and K. Li, "A dynamic automated lane change maneuver based on vehicle-to-vehicle communication," Transportation Research Part C: Emerging Technologies, vol. 62, pp. 87-102, 2016.

[4] F. You, R. Zhang, G. Lie, H. Wang, H. Wen, and J. Xu, "Trajectory planning and tracking control for autonomous lane change maneuver based on the cooperative vehicle infrastructure system," Expert Systems with Applications, vol. 42, no. 14, pp. 5932-5946, 2015.

[5] D. Yang, S. Zheng, C. Wen, P. J. Jin, and B. Ran, "A dynamic lanechanging trajectory planning model for automated vehicles," Transportation Research Part C: Emerging Technologies, vol. 95, pp. 228-247, 2018.

[6] I. A. Ntousakis, I. K. Nikolos, and M. Papageorgiou, "Optimal vehicle trajectory planning in the context of cooperative merging on highways," Transportation research part C: emerging technologies, vol. 71, pp. 464488,2016
[7] W. Cao, M. Mukai, T. Kawabe, H. Nishira, and N. Fujiki, "Cooperative vehicle path generation during merging using model predictive control with real-time optimization," Control Engineering Practice, vol. 34, pp. 98-105, 2015.

[8] C. Xiu and H. Chen, "A behavior-based path planning for autonomous vehicle," in International Conference on Intelligent Robotics and Applications, pp. 1-9, Springer, 2010.

[9] P. Zhao, J. Chen, T. Mei, and H. Liang, "Dynamic motion planning for autonomous vehicle in unknown environments," in 2011 IEEE Intelligent Vehicles Symposium (IV), pp. 284-289, IEEE, 2011.

[10] L. Ma, J. Yang, and M. Zhang, "A two-level path planning method for on-road autonomous driving," in 2012 second international conference on intelligent system design and engineering application, pp. 661-664, IEEE, 2012.

[11] A. Broggi, P. Medici, P. Zani, A. Coati, and M. Panciroli, "Autonomous vehicles control in the vislab intercontinental autonomous challenge," Annual Reviews in Control, vol. 36, no. 1, pp. 161-171, 2012.

[12] M. McNaughton, C. Urmson, J. M. Dolan, and J.-W. Lee, "Motion planning for autonomous driving with a conformal spatiotemporal lattice," in 2011 IEEE International Conference on Robotics and Automation, pp. 4889-4895, IEEE, 2011.

[13] M. Wang, T. Ganjineh, and R. Rojas, "Action annotated trajectory generation for autonomous maneuvers on structured road networks," in The 5 th International conference on automation, robotics and applications, pp. 67-72, IEEE, 2011.

[14] J. Hardy and M. Campbell, "Contingency planning over probabilistic obstacle predictions for autonomous road vehicles," IEEE Transactions on Robotics, vol. 29, no. 4, pp. 913-929, 2013.

[15] H. Sun, W. Deng, S. Zhang, S. Wang, and Y. Zhang, "Trajectory planning for vehicle autonomous driving with uncertainties," in Proceedings 2014 International Conference on Informative and Cybernetics for Computational Social Systems (ICCSS), pp. 34-38, IEEE, 2014.

[16] C. Katrakazas, M. Quddus, W.-H. Chen, and L. Deka, "Real-time motion planning methods for autonomous on-road driving: State-of-the-art and future research directions," Transportation Research Part C: Emerging Technologies, vol. 60, pp. 416-442, 2015.

[17] J. Nilsson, M. Brännström, E. Coelingh, and J. Fredriksson, "Longitudinal and lateral control for automated lane change maneuvers," in 2015 American Control Conference (ACC), pp. 1399-1404, IEEE, 2015.

[18] J. Nilsson, M. Brännström, E. Coelingh, and J. Fredriksson, "Lane change maneuvers for automated vehicles," IEEE Transactions on Intelligent Transportation Systems, vol. 18, no. 5, pp. 1087-1096, 2016.

[19] J. Nilsson, J. Silvlin, M. Brannstrom, E. Coelingh, and J. Fredriksson, "If, when, and how to perform lane change maneuvers on highways," IEEE Intelligent Transportation Systems Magazine, vol. 8, no. 4, pp. 6878, 2016.

[20] J. Kong, M. Pfeiffer, G. Schildbach, and F. Borrelli, "Kinematic and dynamic vehicle models for autonomous driving control design," in 2015 IEEE Intelligent Vehicles Symposium (IV), pp. 1094-1099, IEEE, 2015.

[21] S. Shen, J. Wang, P. Shi, and G. Premier, "Nonlinear dynamics and stability analysis of vehicle plane motions," Vehicle System Dynamics, vol. 45 , no. 1 , pp. $15-35,2007$.

[22] A. Kanaris, E. B. Kosmatopoulos, and P. A. Loannou, "Strategies and spacing requirements for lane changing and merging in automated highway systems," IEEE transactions on vehicular technology, vol. 50, no. 6, pp. 1568-1581, 2001.

[23] D. J. Sun and L. Elefteriadou, "Lane-changing behavior on urban streets: A focus group-based study," Applied ergonomics, vol. 42, no. 5, pp. 682691, 2011

[24] M. Rahman, M. Chowdhury, Y. Xie, and Y. He, "Review of microscopic lane-changing models and future research opportunities," IEEE transactions on intelligent transportation systems, vol. 14, no. 4, pp. 1942-1956, 2013.

[25] T. Lajunen, D. Parker, and S. G. Stradling, "Dimensions of driver anger, aggressive and highway code violations and their mediation by safety orientation in uk drivers," Transportation Research Part F: Traffic Psychology and Behaviour, vol. 1, no. 2, pp. 107-121, 1998. 